Article

\title{
On-Board State-of-Health Estimation at a Wide Ambient Temperature Range in Lithium-Ion Batteries
}

\section{Tiansi Wang, Lei Pei *, Tingting Wang, Rengui Lu and Chunbo Zhu *}

School of Electrical Engineering and Automation, Harbin Institute of Technology, Harbin 150001, China; E-Mails: tiansi.wang@hotmail.com (T.W.); wuyu2012331@163.com (T.W.); lurengui@hit.edu.cn (R.L.)

* Authors to whom correspondence should be addressed; E-Mails: lei.pei@hotmail.com (L.P.); zhuchunbo@hit.edu.cn (C.Z.); Tel./Fax: +86-451-8641-3621 (L.P. \& C.Z.).

Academic Editor: Haolin Tang

Received: 18 June 2015 / Accepted: 3 August 2015 / Published: 11 August 2015

\begin{abstract}
A state-of-health ( $\mathrm{SOH}$ ) estimation method for electric vehicles (EVs) is presented with three main advantages: (1) it provides joint estimation of cell's aging states in terms of power and energy (i.e., $\mathrm{SOHP}$ and $\mathrm{SOH}$ ) - because the determination of SOHP and $\mathrm{SOH}$ e can be reduced to the estimation of the ohmic resistance increase and capacity loss, respectively, the ohmic resistance at nominal temperature will be taken as a health indicator, and the capacity loss is estimated based on a mechanistic model that is developed to describe the correlation between resistance increase and capacity loss; (2) it has wide applicability to various ambient temperatures - to eliminate the effects of temperature on the resistance, another mechanistic model about the resistance against temperature is presented, which can normalize the resistance at various temperatures to its standard value at the nominal temperature; and (3) it needs low computational efforts for on-board application-based on a linear equation of cell's dynamic behaviors, the recursive least-squares (RLS) algorithm is used for the resistance estimation. Based on the designed performance and validation experiments, respectively, the coefficients of the models are determined and the accuracy of the proposed method is verified. The results at different aging states and temperatures show good accuracy and reliability.
\end{abstract}

Keywords: lithium-ion batteries; state-of-health ( $\mathrm{SOH})$; wide temperature range; resistance increase; capacity loss 


\section{Introduction}

The usage of lithium-ion batteries as a storage system is currently the best choice for portable applications, such as electric vehicles (EVs) and mobile electronics, based on comprehensive consideration of its energy and power density and cycle-life [1]. Unfortunately, with battery aging, its maximum available energy and instantaneous power will gradually fade. In this paper, we will focus on developing an effective estimation technology of the state-of-health ( $\mathrm{SOH}$ ) for lithium-ion batteries in EVs such as battery electric vehicles (BEVs) and hybrid electric vehicles (HEVs).

For EVs, battery $\mathrm{SOH}$ is always defined with two specific forms, and they are $\mathrm{SOH}_{\mathrm{E}}$ and $\mathrm{SOH}$ to indicate the degradation of energy and power, respectively. Based on the simplification processes shown in Equations (1) and (2), respectively, the determination of the battery SOHE and SOHp can be reduced to the estimation of the capacity and ohmic resistance [2]. Wherein, $U_{\text {bat }}$ and $I_{\text {bat }}$ indicate the

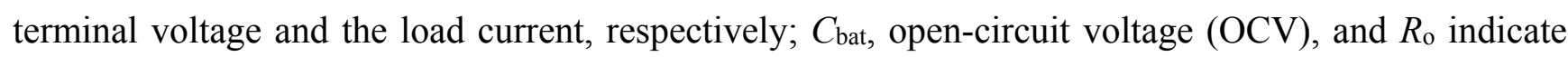
the capacity, open-circuit voltage, and ohmic resistance, respectively; subscripts "a" and " 0 " indicate the aging value and initial value of the marked variables, respectively:

$$
\begin{gathered}
\mathbf{S O H}_{\mathbf{E}}=\frac{\text { Energy }_{\mathrm{a}}}{\text { Energy }_{0}}=\frac{\int_{0}^{t_{\mathrm{a}}} U_{\text {bat }, t} I_{\text {bat }, t} d t}{\int_{0}^{t_{0}} U_{\text {bat }, \mathrm{t}} I_{\text {bat }, t} d t} \approx \frac{\left(U_{\text {bat,max }}-U_{\text {bat,min }}\right) C_{\text {bat,a }}}{\left(U_{\text {bat,max }}-U_{\text {bat,min }}\right) C_{\text {bat }, 0}}=\frac{\boldsymbol{C}_{\text {bat,a }}}{\boldsymbol{C}_{\text {bat }, 0}} \times \mathbf{1 0 0 \%} \\
\mathbf{S O H}_{\mathbf{P}}=\frac{\text { Power }_{\mathrm{a}}}{\text { Power }_{0}} \approx \frac{U_{\text {bat,min }}\left(\mathrm{OCV}-U_{\text {bat,min }}\right) / R_{\mathrm{o}, \mathrm{a}}}{U_{\text {bat,min }}\left(\mathrm{OCV}-U_{\text {bat,min }}\right) / R_{\mathrm{o}, 0}}=\frac{\boldsymbol{R}_{\mathrm{o}, 0}}{\boldsymbol{R}_{\mathrm{o}, \mathrm{a}}} \times \mathbf{1 0 0 \%}
\end{gathered}
$$

For internal resistance identification, the most basic methods are performed by fitting the measured data of the hybrid pulse power characteristic (HPPC) tests or the electrochemical impedance spectroscopy (EIS) tests in least-squares sense under off-line operation conditions [3,4]. In addition, the results of these off-line identifications are always taken as reference values for on-line methods or training data for battery modelling. For the on-line methods, the resistances are always estimated on the basis of equivalent circuit models (ECMs) [5-10] or electrochemical models [11-13] with the utilization of the recursive optimal estimation algorithms, such as the Kalman-filter-based algorithms and the least-squares-based algorithms.

Compared with the resistance identification, the battery capacity cannot be directly identified and will always be calculated by some specific health indicators. In [14-16], the OCV of $\mathrm{LiCO}_{2}$ is selected as the health indicator, and the battery capacity is estimated by a ratio between the changes of coulomb counting and the corresponding changes of state-of-charge (SOC), which is determined by the predefined OCV-SOC relation. However, in most cases, the OCV-SOC relations are not steep enough and always change with aging. For example, the $\mathrm{OCV}$ of $\mathrm{LiFePO}_{4}$ cell shows a flat plateau over a large SOC range due to the two-phase reaction process and the shape of the OCV profile changes obviously at the deep aging stage caused by active material loss. In [17-20], based on the analysis of OCV curve changes, the capacity of $\mathrm{LiFePO}_{4}$ is calculated by an incremental capacity analysis (ICA) to the quasi-OCV curve that is obtained by a discharge process with a tiny current. Besides the OCV, some other parameters can also be used as the indicators. As introduced in [21], the diffusion capacitance of an ECM is taken as the indicator and a correlation between the diffusion capacitance and battery capacity is established. 
For a joint estimation for $\mathrm{SOH}$ and $\mathrm{SOH}$, the internal resistance can be taken as the health indicator, and the $\mathrm{SOH}$ will be estimated by establishing a correlation between the capacity loss and resistance increase [2]. However, the resistance values are very sensitive to temperature changes and the effects of temperature on the resistances are not sufficiently discussed in the existing methods. In addition, the correlation between the capacity loss and resistance increase always lacks of reasonable explanations in terms of chemical and physical meanings.

In this paper, a joint estimation method of $\mathrm{SOH}$ and $\mathrm{SOH}_{\mathrm{E}}$ for EVs over a wide temperature range is proposed with a health indicator of battery ohmic resistance. In Section 2, two mechanistic models are established to describe the relationships for the resistance changes against temperatures and capacity loss, respectively, and a systematic estimation procedure is proposed. Based on the experiments designed in Section 3, the coefficients of the models are determined by fitting the data of characteristic experiments, and the accuracy of the new method is verified with the validation experiments in Section 4. Finally, conclusions are drawn in Section 5.

\section{Models and Methods}

\subsection{The Relationship between Resistance Increase and Capacity Loss}

In the aging stage of batteries used in EVs (i.e., capacity loss $<20 \%$ ), the capacity loss ( $\left.Q_{\text {loss }}\right)$ is mainly caused by the loss of lithium inventory with the formation and thickening of the solid electrolyte interphase (SEI) film [4,22], so the capacity loss will be accompanied by the SEI film resistance $\left(R_{\mathrm{SEI}}\right)$ increase.

As studied in $[23,24]$, the SEI film mainly consists of two kinds of components: inorganic and organic substances. If the proportion of inorganic and organic substances within the new SEI film are marked by $m$ and $(1-m)$, and their resistances are marked as $R_{\text {SEI-M }}$ and $R_{\text {SEI-N, respectively, the }}$ correlation between resistance increase and capacity loss can be described as Equation (3). Herein, $R_{\text {SEI-M }}$ and $R_{\text {SEI-N }}$ are proportional to respective capacity loss by default, respectively; the subscript "a" indicates the change caused by aging; and the proportional coefficients of $a_{\mathrm{M}}$ and $a_{\mathrm{N}}$ are the characteristic coefficients dependent on materials:

$$
d R_{\mathrm{SEI}, \mathrm{a}}=d R_{\mathrm{SEI}-\mathrm{M}, \mathrm{a}}+d R_{\mathrm{SEI}-\mathrm{N}, \mathrm{a}}=a_{\mathrm{M}} \times m d Q_{\mathrm{loss}}+a_{\mathrm{N}} \times(1-m) d Q_{\mathrm{loss}}
$$

With the battery aging, the contents of two kinds of the components will change accordingly. On the one hand, with battery aging, SEI film continually grows from the graphite anode surface and its thickness accordingly increases [25,26]. On the other hand, for the SEI film, the dense inner layers close to the graphite are mainly composed of inorganic substances, and the porous outer layers close to the electrolyte mainly consist of organic substances [24,27]. That means, with the capacity loss due to SEI growth, the proportion of organic substances in the new forming SEI film gradually decreases and that of inorganic substances correspondingly increases. When we suppose $m$ is proportional to $Q_{\text {loss }}$ (i.e., $m=b Q_{\mathrm{loss}}$ ), as expressed in Equation (4), the correlation between the $Q_{\text {loss }}$ and $R_{\mathrm{SEI}}$ can be obtained by integrating the Equation (3). Wherein, $a_{1}, a_{2}$ and $C$ are the unknown coefficients in derivation process: 


$$
\begin{aligned}
\int d R_{\mathrm{SEI}, \mathrm{a}} & =\int\left[a_{\mathrm{M}} b Q_{\mathrm{loss}}+a_{\mathrm{N}}\left(1-b Q_{\mathrm{loss}}\right)\right] d Q_{\mathrm{loss}} \\
& \Rightarrow R_{\mathrm{SEI}, \mathrm{a}}=a_{1} Q_{\mathrm{loss}}^{2}+a_{2} Q_{\mathrm{loss}}+C
\end{aligned}
$$

Because a part of SEI film has formed in prior formation cycles before the cells leave the factories, the current SEI film will contain two parts: an initial SEI layer (corresponding to $R_{\mathrm{SEI}, 0}$ and $Q_{\text {loss, }, 0}$ ) and

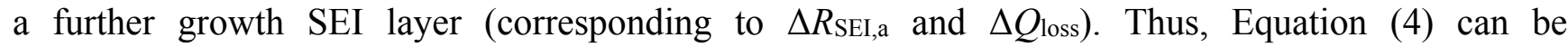
transformed to Equation (5):

$$
\left(\Delta R_{\mathrm{SEI}, \mathrm{a}}+R_{\mathrm{SEI}, 0}\right)=a_{1}\left(\Delta Q_{\mathrm{loss}}+Q_{\mathrm{loss}, 0}\right)^{2}+a_{2}\left(\Delta Q_{\mathrm{loss}}+Q_{\mathrm{loss}, 0}\right)+C
$$

Simplifying Equation (5), we can get the function correlation between the resistance increase and capacity loss as shown in Equation (6):

$$
\Delta R_{\mathrm{SEl,a}}=\alpha_{1} \Delta Q_{\mathrm{loss}}^{2}+\alpha_{2} \Delta Q_{\mathrm{loss}}
$$

However, the $R_{\text {SEI }}$ cannot be independently identified and will be included in the on-line identification result of the $R_{0}$, which is always estimated using the online measured voltage and current data. According to the EIS analysis results presented in [28,29], the $R_{\mathrm{o}}$ mainly corresponds to the internal resistances relevant to fast electrochemical processes, containing the resistances of electrolyte/electrical transport in bulk phase $\left(R_{\text {bulk }}\right)$, SEI film resistance $\left(R_{\text {SEI }}\right)$, and the resistance of faradaic charge transfer at the electrode interphase $\left(R_{\mathrm{ct}}\right)$, and can be approximately expressed as Equation (7):

$$
R_{\mathrm{o}}=R_{\mathrm{bulk}}+R_{\mathrm{SEI}}+R_{\mathrm{ct}}
$$

Because $R_{\text {bulk }}$ and $R_{\text {ct }}$ nearly remain unchanged in this aging stage [28], it can be concluded that the increment of $R_{\mathrm{o}}$ is almost equal to that of $R_{S E I}$, as expressed in Equation (8):

$$
\Delta R_{\mathrm{o}, \mathrm{a}}=\Delta R_{\mathrm{bulk, \textrm {a }}}+\Delta R_{\mathrm{SEl}, \mathrm{a}}+\Delta R_{\mathrm{ct}, \mathrm{a}} \approx \Delta R_{\mathrm{SEI}, \mathrm{a}}
$$

Based on the Equation (8), the function relationship of $\Delta Q_{\text {loss }}$ with respect to $\Delta R_{\mathrm{o}, \mathrm{a}}$, which is the inverse function of Equation (6), can be expressed by Equation (9). Wherein, the coefficients $\alpha_{1}$ and $\alpha_{2}$ will be determined by experimental data:

$$
\Delta Q_{\mathrm{loss}}=\frac{-\alpha_{2}+\sqrt{\alpha_{2}^{2}+4 \alpha_{1} \Delta R_{\mathrm{SEI}, \mathrm{a}}}}{2 \alpha_{1}} \approx \frac{-\alpha_{2}+\sqrt{\alpha_{2}^{2}+4 \alpha_{1} \Delta R_{\mathrm{o}, \mathrm{a}}}}{2 \alpha_{1}}
$$

\subsection{The Effects of Temperature on Internal Resistances}

The effects of temperature on different kinds of internal resistances are different, so different internal resistances may have different changing trends. For the bulk and SEI resistance (i.e., Rbulk and $\left.R_{\mathrm{SEI}}\right)$, the resistance increment is a near linear function of temperature change; however, for the charge transfer resistance (i.e., $R_{\mathrm{ct}}$ ), the resistance value is inversely proportional to the electrochemical reaction rate and the reaction rate follows an Arrhenius dependence with temperature (T) [30]. Therefore, the correlation between the ohmic resistance change $\left(\Delta R_{0}, t\right)$ and $T$ can be deduced as Equation (10). Wherein, the subscript " $t$ " indicates the change caused by ambient temperatures; 
the coefficients $\kappa_{1}-\kappa_{4}$ will be determined by experimental data; and $T_{\text {std }}$ indicates the nominal temperature which is set to $30{ }^{\circ} \mathrm{C}$ in this paper:

$$
\begin{aligned}
\Delta R_{\mathrm{o}, t} & =\Delta R_{\mathrm{bulk}, t}+\Delta R_{\mathrm{SEI}, t}+\Delta R_{\mathrm{ct}, t} \\
& =k_{\mathrm{bulk}}\left(T-T_{\mathrm{std}}\right)+k_{\mathrm{SEI}-\mathrm{M}}\left(T-T_{\mathrm{std}}\right)+k_{\mathrm{SEI}-\mathrm{N}}\left(T-T_{\mathrm{std}}\right)+k_{\mathrm{ct}}\left(\frac{1}{e^{-E_{\mathrm{a}} /(T+273)}}-\frac{1}{e^{-E_{\mathrm{a}} /\left(T_{\mathrm{std}}+273\right)}}\right) \\
& =\kappa_{1} T+\kappa_{2} e^{\kappa_{3} /(T+273)}+\kappa_{4}
\end{aligned}
$$

This new mechanistic model can be used to eliminate the effects of temperatures on the ohmic resistance, which ensures that the $\mathrm{SOH}$ estimation can be used over a wide temperature range in practical operations.

\subsection{The Parameter Identification Method}

As shown in Figure 1, an ECM employing a resistance in series with a parallel resistance and capacitance (R-RC), which is proved to be the best choice for pursuing the balance between the fitting effects and the computational efforts and most widely applied for battery state estimations [31,32], is chosen for on-line resistance identification in this study. Wherein, the OCV is used to express the internal voltage source of the battery model; $R_{\mathrm{o}}$ and $R_{\mathrm{p}}$ denote the ohmic resistance and polarization resistance, respectively; and $C_{\mathrm{p}}$ is the polarization capacitance.

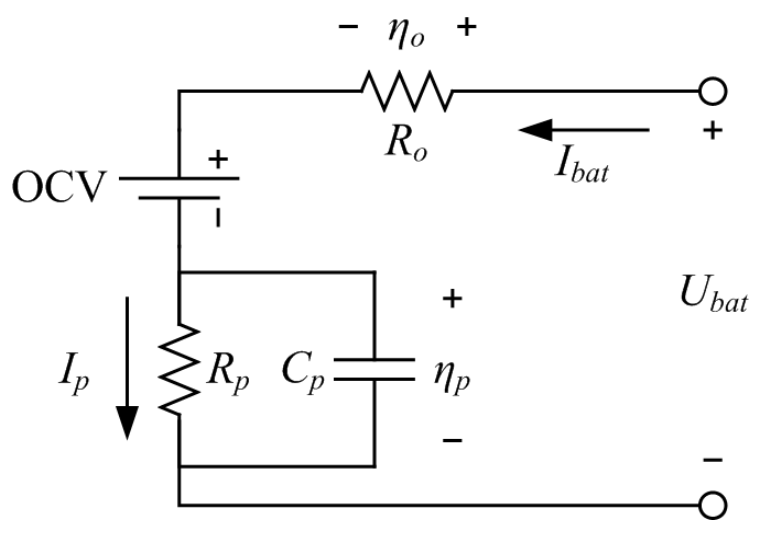

Figure 1. A resistance in series with a parallel resistance and capacitance (R-RC) model.

The on-line internal resistance identification method requires determination with two steps:

First, based on the chosen ECM, the terminal voltage of a cell can be expressed as Equation (11) [33]. Herein, the subscript " $k$ " represents $k$ th time step, and $\tau_{\mathrm{p}}=R_{\mathrm{p}} C_{\mathrm{p}}$.

$$
U_{\text {bat }, k-1}=\mathrm{OCV}+\left(R_{\mathrm{o}}+R_{\mathrm{p}}\right) I_{\text {bat }, k-1}+R_{\mathrm{o}} \tau_{\mathrm{p}} \frac{I_{\text {bat }, k}-I_{\text {bat }, k-1}}{\Delta t}+\tau_{\mathrm{p}} \frac{U_{\text {bat }, k-1}-U_{\text {bat }, k}}{\Delta t}
$$

Separating the known and unknown variables of Equation (11) and rearrangement yield a linear expression about the parameter vector, as shown in Equation (12). Wherein, $\mathrm{z} k$ and $\varphi k$ are obtained by the measured data of the current and voltage at the sample points; and $\theta_{k}=\left[\mathrm{OCV}, R_{\mathrm{o}, k}+R_{\mathrm{p}, k}, R_{\mathrm{o}, k} \tau_{\mathrm{p}, k}, \tau_{\mathrm{p}, k}\right]^{\mathrm{T}}$ is the target vector to be determined: 


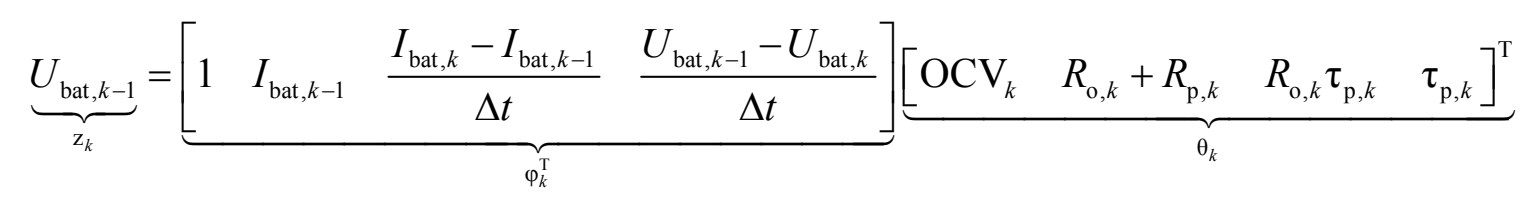

Second, to identify the parameters in real time, a discrete-time filter is always needed. The recursive least-squares (RLS) algorithm with a forgetting factor is adopted in this study, which is very suitable for on-board applications due to its low computational efforts and memory consumptions. Refer to [34,35] for details of the RLS method and its generalization. The implementation of RLS algorithm with a forgetting factor is detailed in Table 1 . Herein, $P_{k}$ represents an estimation error covariance matrix; $K_{k}$ indicates a gain matrix; and ${ }^{\wedge}$ represents an estimation value.

Following the procedure in Table 1 , the target vector $\theta_{k}$ can be easily determined, and the identification results of the parameters can be calculated as follows:

(1) Initial the target vector $\theta_{0}$ and the covariance matrix $P_{0}$;

(2) For $k=1,2,3, \ldots$, after new measurements, $\mathrm{z}_{k}$ and $\varphi_{k}$ are available;

(3) Update $\theta_{k}$ and $P_{k}$ with the equations in Table 1;

(4) Calculate the parameter values with Equation (13).

$$
\left\{\begin{aligned}
\mathrm{OCV}_{k} & =\theta_{k}(1) \\
R_{\mathrm{o}, k} & =\theta_{k}(3) / \theta_{k}(4) \\
R_{\mathrm{p}, k} & =\theta_{k}(2)-\theta_{k}(3) / \theta_{k}(4) \\
\tau_{\mathrm{p}, k} & =\theta_{k}(4)
\end{aligned}\right.
$$

Table 1. Procedures of the recursive least-squares (RLS) for parameter identification.

\begin{tabular}{cc}
\hline Initializations $^{\text {a }}$ & Updates $^{\mathbf{b}}$ \\
\hline & $K_{k}=P_{k-1} \varphi_{k} /\left(\lambda+\varphi_{k}^{\mathrm{T}} P_{k-1} \varphi_{k}\right)$ \\
$\hat{\theta}_{0}=0, P_{0}=\delta^{-1} \mathrm{I}$ & $\hat{\theta}_{k}=\hat{\theta}_{k-1}+K_{k}\left(z_{k}-\hat{\theta}_{k-1}^{\mathrm{T}} \varphi_{k}\right)$ \\
& $P_{k}=\left(\mathrm{I}-K_{k} \varphi_{k}^{\mathrm{T}}\right) P_{k-1} / \lambda$ \\
\hline
\end{tabular}

${ }^{\mathrm{a}} \delta$ is set to $10^{-3} ;{ }^{\mathrm{b}} \lambda$ is a forgetting factor.

\subsection{State of Health (SOH) Estimation Procedure}

Based on the new models and parameter identification method presented above, a new $\mathrm{SOH}$ estimation procedure is shown in Figure 2. 


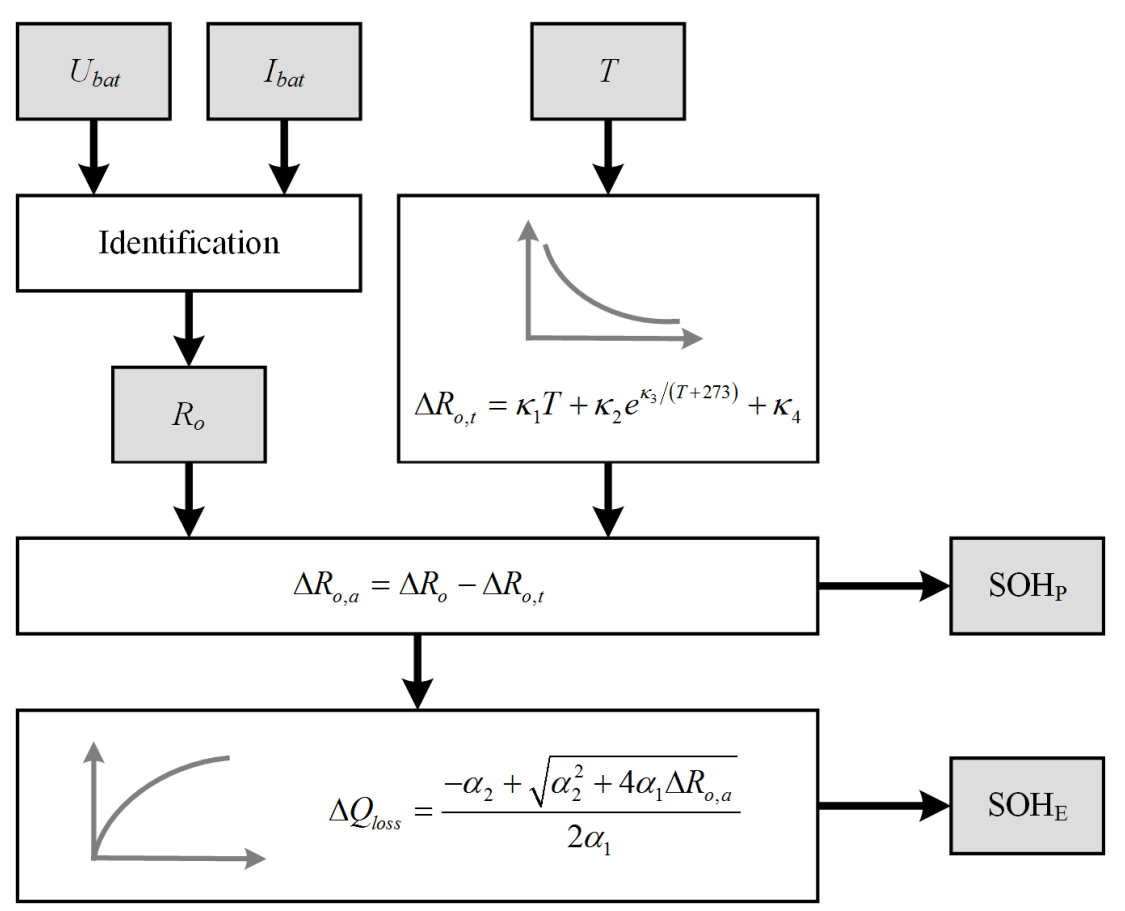

Figure 2. The procedure of the State of Health $(\mathrm{SOH})$ estimation method.

The procedure is listed as follows: (1) on-line ohmic resistance identification: through the measurements of battery voltage and current, the ohmic resistance values can be identified on line using the RLS algorithm; (2) SOHP estimation: according to the measured ambient temperature, the ohmic resistance increment caused by various temperatures can be calculated by Equation (10), and then this increment is subtracted from the identified resistance, which is a resistance normalization process, so the normalized resistance can be used to reflect battery aging levels and estimate SOHP by Equation (2); (3) SOHE estimation: the capacity loss can be obtained by substitution of the normalized $R_{\mathrm{o}}$ increment into Equation (9), and then the $\mathrm{SOH}_{\mathrm{E}}$ can be calculated according to Equation (1).

\section{Experiment Design}

To determine the coefficients of the mechanistic models and verify the accuracy of the estimation method, the experiments are designed as follows.

\subsection{Experiment Object}

In this research, 32650-type $\mathrm{LiFePO}_{4} /$ graphite cells (OptimumNano Energy Co. Ltd., Shenzhen, China) with the nominal capacity of $5 \mathrm{Ah}$ were chosen as the research objects, and the upper and lower limit voltages of the chosen cells are $3.65 \mathrm{~V}$ and $2.5 \mathrm{~V}$, respectively.

\subsection{Experiment Procedure}

In this study, the experiments consist of two main parts: the aging cycles and the performance tests. In addition, the performance tests can be subdivided into the characteristic tests and the validation tests, as shown in Figure 3. 


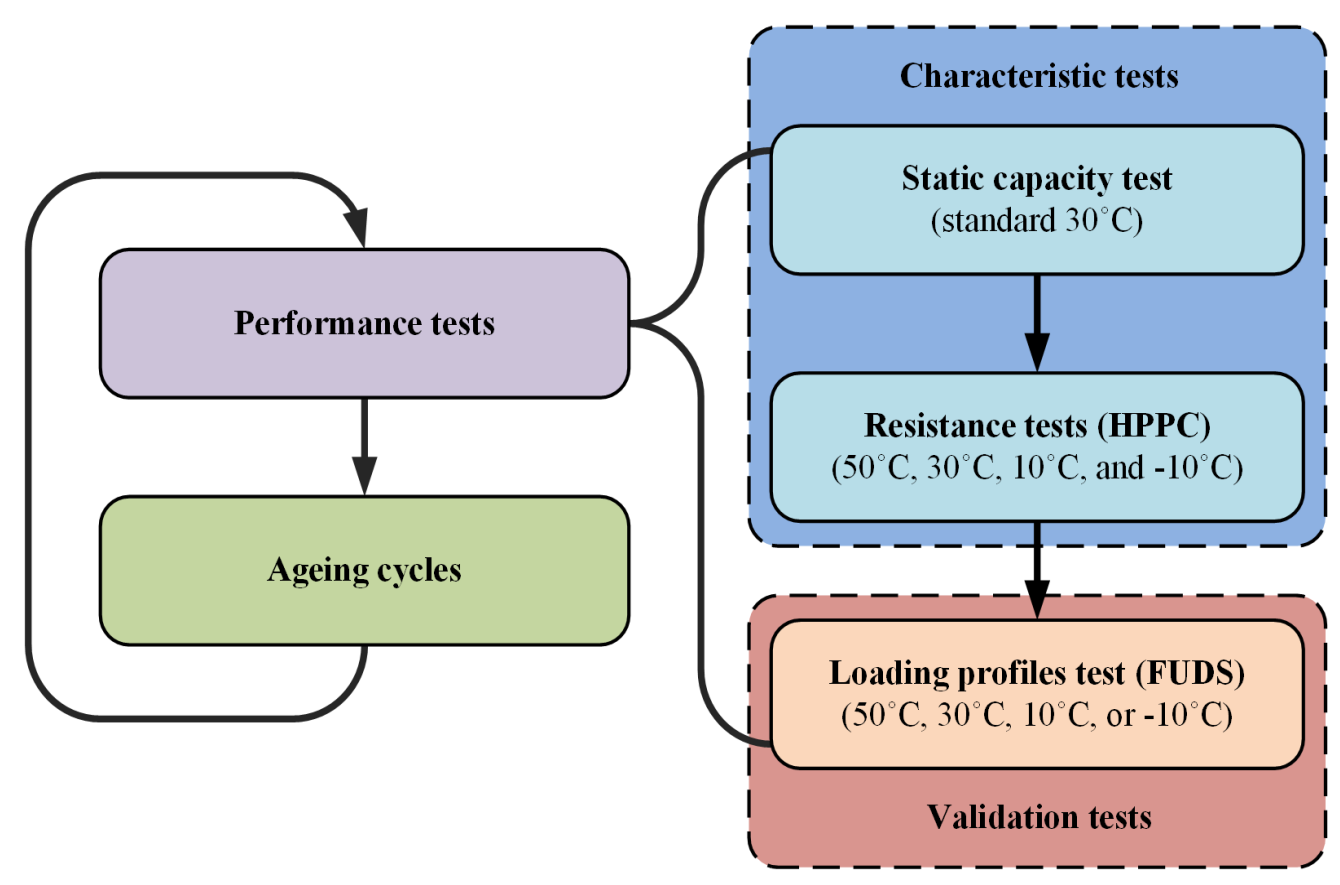

Figure 3. The procedure of experiments.

To shorten the period of the aging experiments, the aging cycles were performed at the ambient temperature of $60{ }^{\circ} \mathrm{C}$ with the current of $1 \mathrm{C}$-rate. The performance tests were performed every 100 aging cycles, which contain three parts: a static capacity test, an internal resistance test, and a validation test. Herein, the capacity test was performed with a $\mathrm{CC}-\mathrm{CV}$ (constant current-constant voltage) charge and a constant discharge with the current of $1 \mathrm{C}$-rate at a normal temperature of $30{ }^{\circ} \mathrm{C}$; the HPPC tests, as the resistance tests, were conducted at four different temperatures (in the order of $50{ }^{\circ} \mathrm{C}, 30{ }^{\circ} \mathrm{C}, 10{ }^{\circ} \mathrm{C}$, and $-10{ }^{\circ} \mathrm{C}$ ); and Federal Urban Driving Schedule (FUDS) tests taken as validation experiments were performed at set temperatures of $50{ }^{\circ} \mathrm{C}, 30{ }^{\circ} \mathrm{C}, 10{ }^{\circ} \mathrm{C}$, and $-10{ }^{\circ} \mathrm{C}$, respectively.

\subsection{Experiment Equipment}

All the tests were performed with a channel of the Arbin instruments' BT2000 test bench (Arbin Instruments Co. Ltd., College Station, TX, USA), which has a voltage measurement accuracy of $\pm 0.01 \%$ and a current measurement accuracy of $\pm 0.02 \%$ on the full-scale value of both ranges $(18 \mathrm{~V}, \pm 10 \mathrm{~A}$ for the medium current range and $\pm 100 \mathrm{~A}$ for the high current range). Moreover, the cell ambient temperatures were controlled within $\pm 2{ }^{\circ} \mathrm{C}$ by incubators in all tests.

\section{Results and Discussion}

\subsection{Performance Test Results}

The capacity values of the battery obtained by the static capacity tests are shown as follows in Table 2. 
Table 2. Cell capacity and capacity loss at different aging states.

\begin{tabular}{ccc}
\hline Aging state & Capacity $(\mathbf{A h})$ & $\boldsymbol{Q}_{\text {loss }}(\mathbf{A h})$ \\
\hline Fresh & 4.992 & 0 \\
100 cycles & 4.762 & 0.23 \\
200 cycles & 4.378 & 0.614 \\
300 cycles & 3.754 & 1.238 \\
\hline
\end{tabular}

The reference values of ohmic resistance are identified with the least-squares method applied to the voltage and current data from the pulse current experiments of HPPC tests, and the results at different aging levels and temperatures are shown in Figure 4. For brevity, the application process of the least-squares fit is omitted here.

From Figure 4, it can be observed that the $R_{o}$ values increase with the battery aging and are nearly identical in the SOC range from 0.4 to 0.6 . Thus, the $R_{o}$ values at around SOC $=0.5$ are taken as the indicators. This selection not only ensures the resistance values are hardly affected by the SOC estimation errors at an acceptable level (e.g., less than $\pm 5 \%$ ) but also makes it possible to implement the algorithm in different types of EVs (e.g., general SOC ranges of 0.2-1 for BEVs and 0.3-0.7 for HEVs).
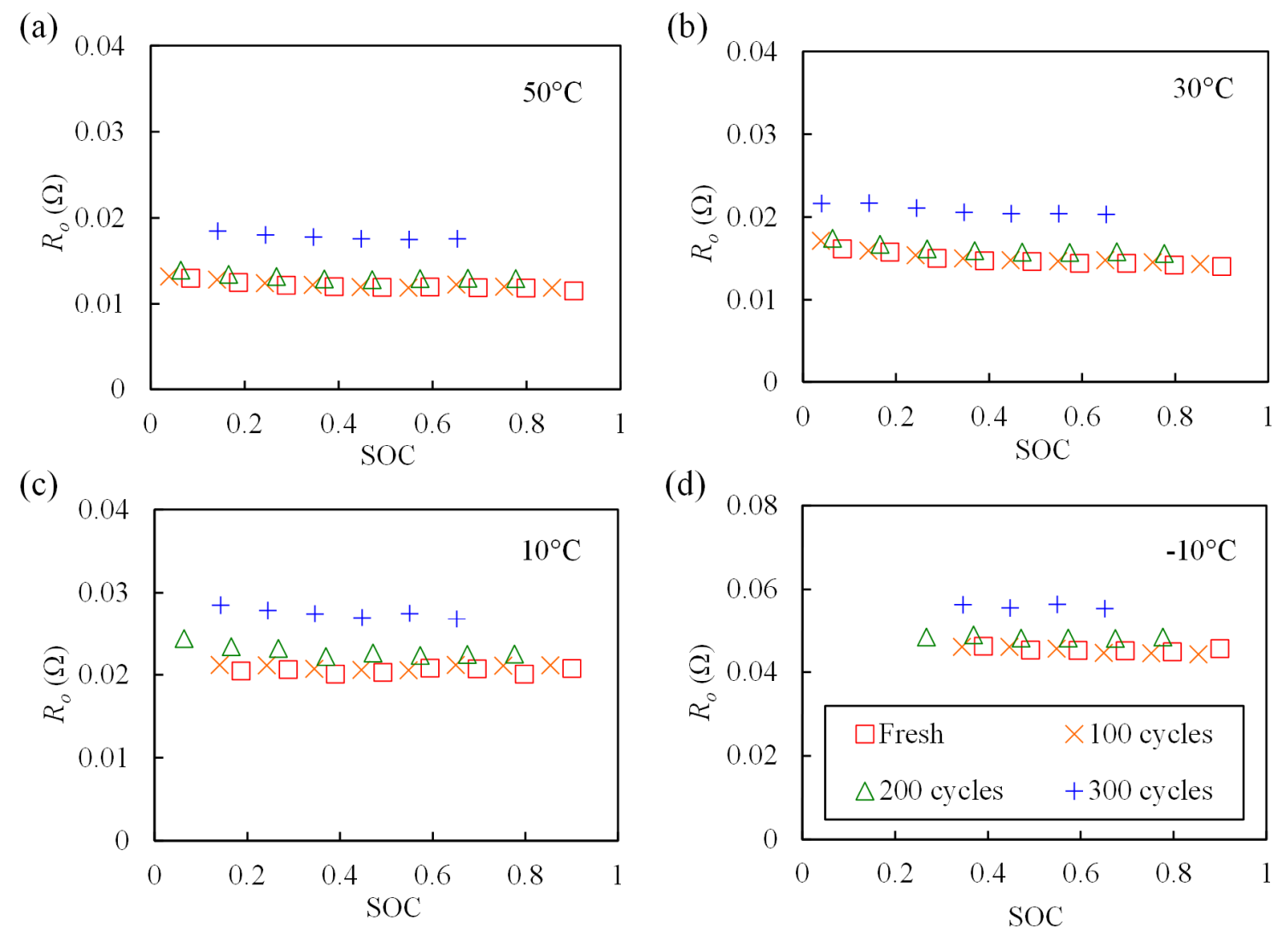

Figure 4. $R_{\mathrm{o}}$ values of different aging cycles at various temperatures: (a) $50{ }^{\circ} \mathrm{C}$; (b) $30{ }^{\circ} \mathrm{C}$; (c) $10{ }^{\circ} \mathrm{C} ;(\mathbf{d})-10{ }^{\circ} \mathrm{C}$.

The values of $R_{\mathrm{o}}$ at around $\mathrm{SOC}=0.5$ are plotted against different temperatures and aging states in Figure 5. From this figure, it can be observed that $R_{\mathrm{o}}$ increases with battery ages and temperature decreases. To verify the reliability of the mechanistic models and determine the coefficients of the 
proposed models, the specific correlations of $R_{o}$ increment with respect to capacity loss $\left(\Delta R_{\mathrm{o}, \mathrm{a}} v s . Q_{\text {loss }}\right)$ and temperature $\left(\Delta R_{\mathrm{o}, t} v s . T\right)$ will be further investigated in the next subsection.

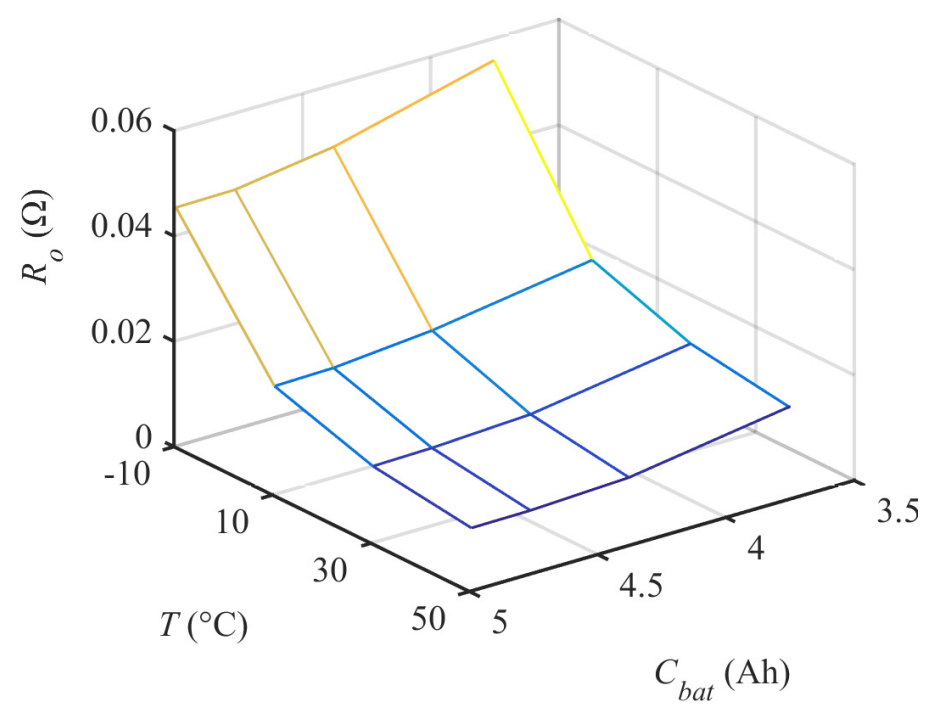

Figure 5. $R_{\mathrm{o}}$ values with different temperatures at different aging levels.

\subsection{Model Coefficient Determining}

First, as a part of Figure 5, the capacity loss is plotted against resistance increase at the nominal temperature by discrete points in Figure 6. Based on these discrete data, the coefficients of Equation (9) are fitted with the least-squares method and the fitting results are given as Equation (14):

$$
\Delta Q_{\text {loss }}=\frac{-\alpha_{2}+\sqrt{\alpha_{2}^{2}+4 \alpha_{1} \Delta R_{\mathrm{o}, \mathrm{a}}}}{2 \alpha_{1}}, \text { where }\left\{\begin{array}{l}
\alpha_{1}=4.154 \times 10^{-3} \\
\alpha_{2}=2.623 \times 10^{-4}
\end{array}\right.
$$

Additionally, the fitted curve is also plotted in Figure 6. From the fitting results, it can be observed that the experiment results are highly consistent with the predictions of the mechanistic model for the relation between capacity loss and resistance increase (i.e., Equation (9)). Wherein, the small fitting errors are probably caused by the simplification to the complex components of SEI film.

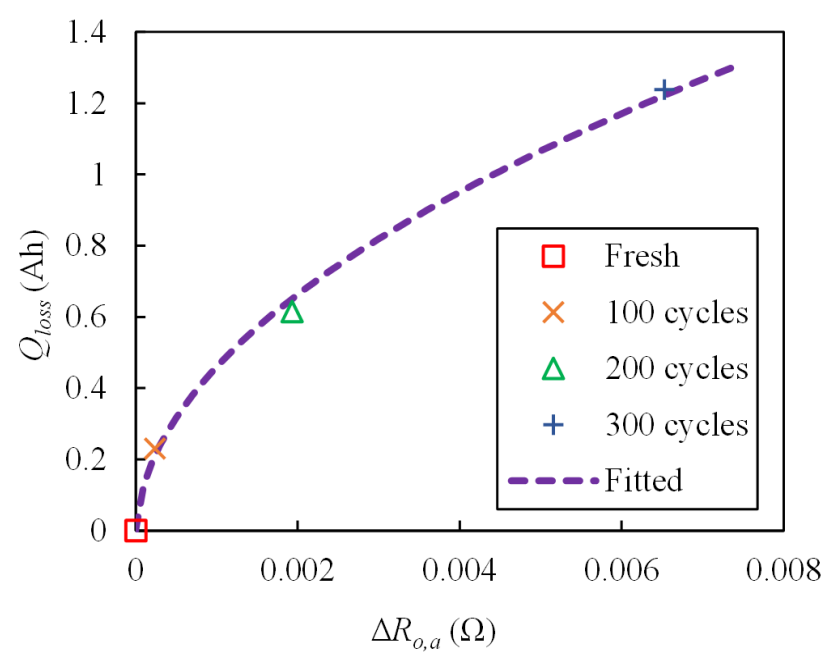

Figure 6. The relationship between $\Delta R_{\mathrm{o}, \mathrm{a}}$ and $Q_{\text {loss. }}$ 
Second, the relationship between the ohmic resistance increment (relative to the nominal value at $30{ }^{\circ} \mathrm{C}$ ) and temperature is plotted in Figure 7 with discrete points.

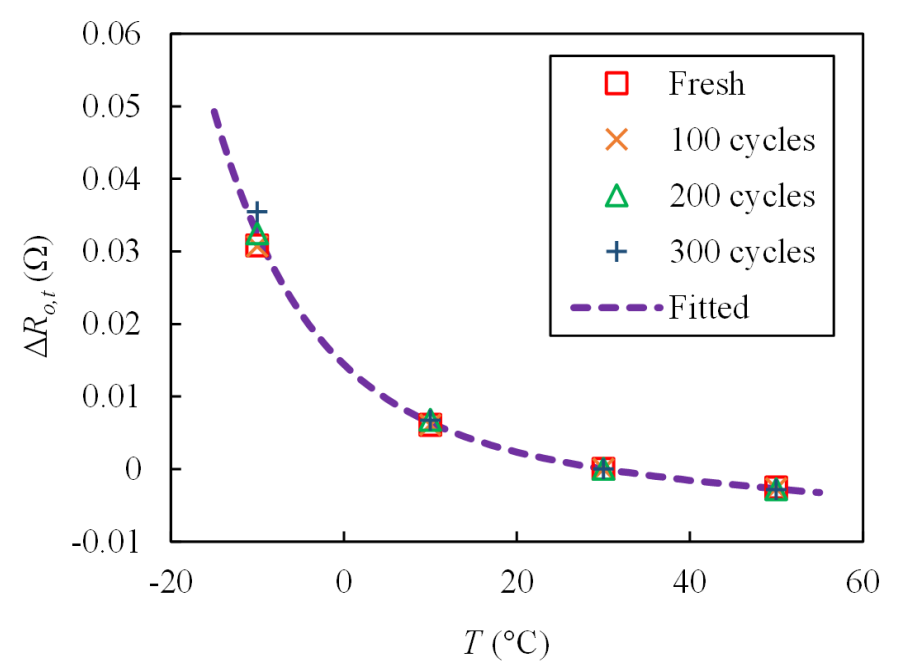

Figure 7. The relationship of $\Delta R_{\mathrm{o}, t}$ with temperature.

The unknown coefficients of Equation (10) are determined by a least-squares fit of these discrete data, and the results are shown as Equation (15). The fitted curve is also plotted in Figure 7 and shows that the incremental changes in ohmic resistance along temperatures follow an exponential relationship, which agrees well with the predictions of the mechanistic model reflecting the temperature effects.

$$
\Delta R_{\mathrm{o}, t}=\kappa_{1} T+\kappa_{2} e^{\kappa_{3} /(T+273)}+\kappa_{4}, \text { where }\left\{\begin{array}{l}
\kappa_{1}=7.602 \times 10^{-12} \\
\kappa_{2}=5.818 \times 10^{3} \\
\kappa_{3}=2.236 \times 10^{-2} \\
\kappa_{4}=-7.928 \times 10^{-5}
\end{array}\right.
$$

\subsection{Validating Results}

In consideration of the effects of ambient temperatures on the estimation algorithm, the FUDS working condition experiments, which were performed on the cells under four different conditions of fresh state $/ 50{ }^{\circ} \mathrm{C}, 100$ aging cycles $/ 30{ }^{\circ} \mathrm{C}, 200$ aging cycles $/ 10{ }^{\circ} \mathrm{C}$, and 300 aging cycles $/-10{ }^{\circ} \mathrm{C}$, are chosen to verify the estimation accuracy of the algorithm. Based on the collected data of terminal voltage and load current, the ohmic resistance is identified by applying the RLS algorithm with a forgetting factor to Equation (12) with the initial values of $\theta_{0}=\left[\begin{array}{llll}0 & 0 & 0 & 0\end{array}\right]^{\mathrm{T}}$ and the forgetting factor $\lambda=0.999$. The identified results are shown by solid curves in Figure 8, and compared with the reference values obtained from off-line HPPC tests. 


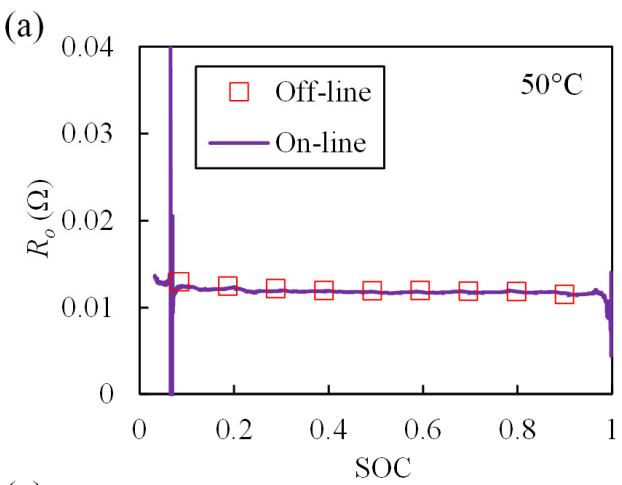

(c)

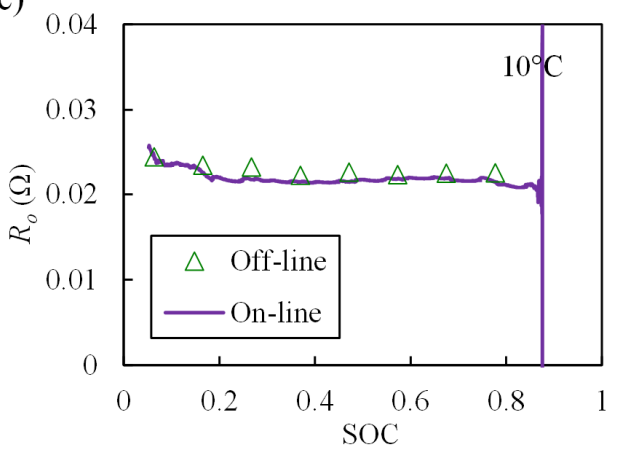

(b)

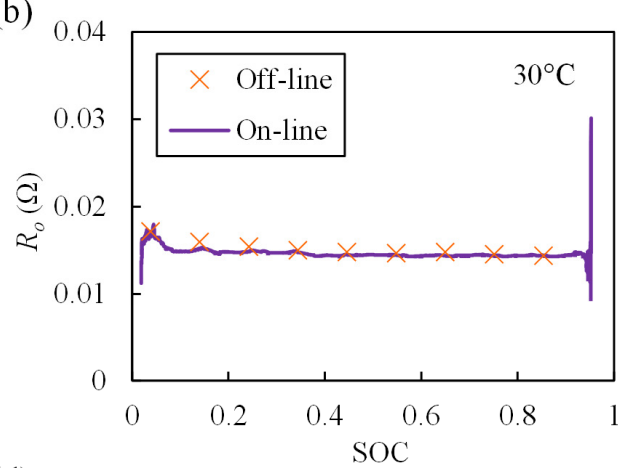

(d)

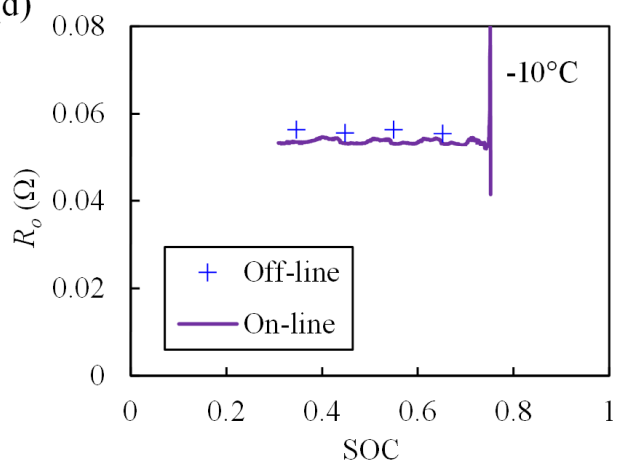

Figure 8. The comparison of the identification and reference values of $R_{\mathrm{o}}$ : (a) fresh values at $50{ }^{\circ} \mathrm{C}$; (b) the values after 100 cycles at $30{ }^{\circ} \mathrm{C}$; (c) the values after 200 cycles at $10{ }^{\circ} \mathrm{C}$; (d) the values after 300 cycles at $-10^{\circ} \mathrm{C}$.

From Figure 8, it can be found that there are only small differences between the identified results and the reference values in all four tests, suggesting a good robustness of the identification method at different temperatures and aging levels. Wherein, the estimated $R_{\mathrm{o}}$ values are always slightly less than the reference values of the HPPC tests, probably because the cell's inner temperature will be slightly higher than the ambient temperature under continuous operation conditions, especially for a lower ambient temperature.

Based on the ohmic resistance identification results, the $\mathrm{SOHp}$ and $\mathrm{SOHE}$ estimation results are obtained by following the procedure presented in Section 2.4, and are shown in Table 3. Table 3 shows that the on-line $\mathrm{SOH}$ and $\mathrm{SOH}_{\mathrm{E}}$ estimation errors of the new method are less than $\pm 5 \%$ at various ambient temperatures and aging states, which indicates that the proposed approach has good accuracy and reliability for on-board application.

Table 3. Validation results for state-of-health (SOHp and $\mathrm{SOH} E$ ).

\begin{tabular}{cccccc}
\hline $\begin{array}{c}\text { Aging } \\
\text { cycles }\end{array}$ & $\begin{array}{c}\text { Temperature } \\
\left({ }^{\mathbf{C}} \mathbf{C}\right)\end{array}$ & $\begin{array}{c}\text { Measured } \\
\mathbf{S O H}_{\mathbf{P}}(\mathbf{\%})\end{array}$ & $\begin{array}{c}\text { Estimated } \\
\mathbf{S O H}_{\mathbf{P}} \mathbf{( \% )}\end{array}$ & $\begin{array}{c}\text { Measured } \\
\mathbf{S O H}_{\mathbf{E}}(\mathbf{\%})\end{array}$ & $\begin{array}{c}\text { Estimated } \\
\mathbf{S O H}_{\mathbf{E}}(\mathbf{\%})\end{array}$ \\
\hline 0 & 50 & 100.0 & 100.6 & 100.0 & 100.6 \\
100 & 30 & 98.4 & 99.8 & 95.4 & 98.7 \\
200 & 10 & 92.2 & 94.7 & 87.7 & 91.8 \\
300 & -10 & 71.3 & 70.9 & 75.2 & 76.7 \\
\hline
\end{tabular}

\section{Conclusions}

This paper proposes an on-line $\mathrm{SOH}$ estimation method for lithium-ion batteries applied in EVs. The presented SOH estimation method has three characteristics: (1) joint estimation of SOHP and 
SOHE; (2) eliminating ambient temperature effects; and (3) low computational efforts for on-board application. In this study, the establishment of two mechanistic models is confirmed to be reliable and accurate by the battery characteristic tests, and the coefficients of the models are determined by a curve fit. Additionally, the proposed method has been verified by the validation experiments, and the results show good accuracy and wide applicability at various ambient temperatures.

\section{Acknowledgments}

This research was supported in part by an International Cooperation and Exchange Program between the National Natural Science Funds of China and the UK Engineering and Physical Sciences Research Council (NSFC: 51361130153, EPSRC: EP/L001063/1), in part by the Research and Development of Application Technology Plan Project in Heilongjiang Province of China (GA13A202), and in part by the Science and Technology Project of the State Grid Corporation of China. The author would also like to thank the reviewers for their corrections and helpful suggestions.

\section{Author Contributions}

Tiansi Wang and Lei Pei cooperated to develop the new method and complete the main parts of the manuscript. Tingting Wang was involved in battery modelling. Rengui Lu and Chunbo Zhu gave some suggestions for the preparation of the manuscript.

\section{Conflicts of Interest}

The authors declare no conflict of interest.

\section{Abbreviations}

BEV battery electric vehicle

ECM equivalent circuit model

EIS electrochemical impedance spectroscopy

EV electric vehicle

FUDS Federal Urban Driving Schedule

HEV hybrid electric vehicle

HPPC hybrid pulse power characterization

ICA incremental capacity analysis

RLS recursive least-squares

$\mathrm{R}-\mathrm{RC}$ a resistance in series with a parallel resistance and capacitance

SEI solid electrolyte interphase

SOC state-of-charge

$\mathrm{SOH}$ state-of-health

\section{References}

1. Scrosati, B.; Garche, J. Lithium batteries: Status, prospects and future. J. Power Sources 2010, $195,2419-2430$. 
2. Waag, W.; Fleischer, C.; Sauer, D.U. Critical review of the methods for monitoring of lithium-ion batteries in electric and hybrid vehicles. J. Power Sources 2014, 258, 321-339.

3. PNGV Battery Test Manual: Revision 3; Technical Report for US Department of Energy: Washington, DC, USA, 2001.

4. Eddahech, A.; Briat, O.; Bertrand, N.; Delétage, J.Y.; Vinassa, J.M. Behavior and state-of-health monitoring of Li-ion batteries using impedance spectroscopy and recurrent neural networks. Int. J. Electr. Power Energy Syst. 2012, 42, 487-494.

5. Plett, G.L. Extended kalman filtering for battery management systems of lipb-based hev battery packs: Part 3. State and parameter estimation. J. Power Sources 2004, 134, 277-292.

6. He, H.; Xiong, R.; Guo, H. Online estimation of model parameters and state-of-charge of $\mathrm{LiFePO}_{4}$ batteries in electric vehicles. Appl. Energy 2012, 89, 413-420.

7. Pei, L.; Zhu, C.; Wang, T.; Lu, R.; Chan, C.C. Online peak power prediction based on a parameter and state estimator for lithium-ion batteries in electric vehicles. Energy 2014, 66, 766-778.

8. Roscher, M.A.; Bohlen, O.S.; Sauer, D.U. Reliable state estimation of multicell lithium-ion battery systems. IEEE Trans. Energy Convers. 2011, 26, 737-743.

9. Xiong, R.; He, H.; Sun, F.; Zhao, K. Online estimation of peak power capability of li-ion batteries in electric vehicles by a hardware-in-loop approach. Energies 2012, 5, 1455-1469.

10. Wang, S.; Verbrugge, M.; Wang, J.S.; Liu, P. Power prediction from a battery state estimator that incorporates diffusion resistance. J. Power Sources 2012, 214, 399-406.

11. Schmidt, A.P.; Bitzer, M.; Imre, Á.W.; Guzzella, L. Model-based distinction and quantification of capacity loss and rate capability fade in Li-ion batteries. J. Power Sources 2010, 195, 7634-7638.

12. Prasad, G.K.; Rahn, C.D. Model based identification of aging parameters in lithium ion batteries. J. Power Sources 2013, 232, 79-85.

13. Samadi, M.F.; Alavi, S.M.M.; Saif, M. Online state and parameter estimation of the Li-ion battery in a bayesian framework. In Proceedings of the American Control Conference (ACC), Washington DC, USA, 17-19 June 2013; pp. 4693-4698.

14. Pop, V.; Bergveld, H.J.; Danilov, D.; Notten, P.P.H.; Regtien, P.P.L. Adaptive state-of-charge indication system for Li-ion battery-powered devices. World Electr. Veh. J. 2007, 1, 38-45.

15. Pop, V.; Bergveld, H.J.; Notten, P.H.L.; Op het Veld, J.H.G.; Regtien, P.P.L. Accuracy analysis of the state-of-charge and remaining run-time determination for lithium-ion batteries. Measurement 2009, 42, 1131-1138.

16. Tang, X.; Mao, X.; Lin, J.; Koch, B. Capacity estimation for Li-ion batteries. In Proceedings of the American Control Conference (ACC), San Francisco, CA, USA, 29 June-1 July 2011.

17. Dubarry, M.; Svoboda, V.; Hwu, R.; Liaw, B.Y. Incremental capacity analysis and close-to-equilibrium OCV measurements to quantify capacity fade in commercial rechargeable lithium batteries. Electrochem. Solid-State Lett. 2006, 9, A454-A457.

18. Zheng, Y.; Lu, L.; Han, X.; Li, J.; Ouyang, M. LiFePO4 battery pack capacity estimation for electric vehicles based on charging cell voltage curve transformation. J. Power Sources 2013, 226, 33-41.

19. Bloom, I.; Jansen, A.N.; Abraham, D.P.; Knuth, J.; Jones, S.A.; Battaglia, V.S.; Henriksen, G.L. Differential voltage analyses of high-power, lithium-ion cells 1 . Technique and application. J. Power Sources 2005, 139, 295-303. 
20. Weng, C.; Cui, Y.; Sun, J.; Peng, H. On-board state of health monitoring of lithium-ion batteries using incremental capacity analysis with support vector regression J. Power Sources 2013, 235, 36-44.

21. Chen, Z.; Mi, C.C.; Fu, Y.; Xu, J.; Gong, X. Online battery state of health estimation based on genetic algorithm for electric and hybrid vehicle applications. J. Power Sources 2013, 240, 184-192.

22. Dubarry, M.; Truchot, C.; Liaw, B.Y.; Gering, K.; Sazhin, S.; Jamison, D.; Michelbacher, C. Evaluation of commercial lithium-ion cells based on composite positive electrode for plug-in hybrid electric vehicle applications. Part II. Degradation mechanism under $2 \mathrm{C}$ cycle aging. J. Power Sources 2011, 196, 10336-10343.

23. Verma, P.; Maire, P.; Novák, P. A review of the features and analyses of the solid electrolyte interphase in Li-ion batteries. Electrochimica Acta 2010, 55, 6332-6341.

24. Aurbach, D. Review of selected electrode-solution interactions which determine the performance of Li and Li ion batteries. J. Power Sources 2000, 89, 206-218.

25. Pinson, M.B.; Bazant, M.Z. Theory of SEI formation in rechargeable batteries: Capacity fade, accelerated aging and lifetime prediction. J. Electrochem. Soc. 2012, 160, A243-A250.

26. Safari, M.; Morcrette, M.; Teyssot, A.; Delacourt, C. Multimodal physics-based aging model for life prediction of Li-ion batteries. J. Electrochem. Soc. 2008, 156, A145-A153.

27. Peled, E.; Golodnitsky, D.; Ardel, G. Advanced model for solid electrolyte interphase electrodes in liquid and polymer electrolytes. J. Electrochem. Soc. 1997, 144, L208-L208.

28. Yoon, S.; Hwang, I.; Lee, C.W.; Ko, H.S.; Han, K.H. Power capability analysis in lithium ion batteries using electrochemical impedance spectroscopy. J. Electroanal. Chem. 2011, 655, 32-38.

29. Li, X.; Wang, T.; Pei, L.; Zhu, C.; Xu, B. A comparative study of sorting methods for lithium-ion batteries. In Proceedings of the Transportation Electrification Asia-Pacific (ITEC Asia-Pacific), 2014 IEEE Conference and Expo, Beijing, China, 31 August-3 September 2014; pp 1-6.

30. Waag, W.; Käbitz, S.; Sauer, D.U. Experimental investigation of the lithium-ion battery impedance characteristic at various conditions and aging states and its influence on the application. Appl. Energy 2013, 102, 885-897.

31. Hu, X.; Li, S.; Peng, H. A comparative study of equivalent circuit models for Li-ion batteries. J. Power Sources 2012, 198, 359-367.

32. He, H.; Xiong, R.; Guo, H.; Li, S. Comparison study on the battery models used for the energy management of batteries in electric vehicles. Energy Convers. Manag. 2012, 64, 113-121.

33. Wang, S.; Verbrugge, M.; Wang, J.S.; Liu, P. Multi-parameter battery state estimator based on the adaptive and direct solution of the governing differential equations. J. Power Sources 2011, 196, 8735-8741.

34. Ljung, L. System Identification: Theory for the User, 2nd ed.; Prentice Hall: Englewod Cliffs, NJ, USA, 1999.

35. Verbrugge, M.; Koch, B. Generalized recursive algorithm for adaptive multiparameter regression application to lead acid, nickel metal hydride, and lithium-ion batteries. J. Electrochem. Soc. 2006, 153, A187-A201.

(C) 2015 by the authors; licensee MDPI, Basel, Switzerland. This article is an open access article distributed under the terms and conditions of the Creative Commons Attribution license (http://creativecommons.org/licenses/by/4.0/). 